\title{
«Si nous ne sommes pas les partenaires compétents pour les questions de santé, qui peut l'être?»
}

A la tête de l'Assemblée des délégués depuis le début de l'année 2014, le Dr Peter Wiedersheim a dirigé avec succès sa première séance en janvier au cours de laquelle il a fait preuve d'habileté en réussissant à accorder suffisamment de temps aux questions et aux discussions malgré un ordre du jour chargé. Nous lui avons demandé comment il conçoit l'avenir de l'AD et son rôle en tant que président. Entretien.

Interview:

Jacqueline Wettstein

Correspondance: Jacqueline Wettstein FMH

Communication Elfenstrasse 18

CH-3000 Berne 15 Tél. 0313591150 Fax 0313591112
Vous dirigez l'Assemblée des délégués depuis le début de l'année. Qu'est-ce qui vous a incité à vous porter candidat? Les encouragements de mes confrères à postuler et aussi mon intérêt pour cette passionnante activité politique. Grâce à ma longue expérience en politique professionnelle et à mon bon réseau, je considère que ma nouvelle fonction représente une chance de mieux intégrer les membres dans la politique de santé. De plus, je souhaite accélérer et optimiser la mise en œuvre des nouveaux modèles de direction de la FMH approuvés à une écrasante majorité par la Chambre médicale le 6 décembre 2007 et aussi améliorer l'impact de la FMH tant à l'interne qu'à l'externe.

Vous avez été l'un des 33 membres de l'AD pendant sept ans. Que retenez-vous de cette expérience? Ce fut passionnant et formateur. Dès la première séance déjà, j'ai pris les rênes du groupe de travail
«Nouveaux modèles de direction de la FMH» et ensuite d'autres tâches intéressantes ont suivi. L'Assemblée des délégués a certes fait preuve de retenue à ses débuts, mais elle a aujourd'hui gagné en assurance.

A mon avis - que d'autres partagent aussi, il s'agit également de travailler à l'amélioration de la compréhension mutuelle. Malgré certaines différences culturelles entre romands et alémaniques, on assiste encore trop souvent à des malentendus d'ordre linguistique. Je souhaite accorder une attention particulière à l'amélioration de la communication car c'est une question d'estime réciproque.

Que souhaiteriez-vous réaliser en tant que président de l'AD?

Je souhaite que le corps médical se montre actif et sûr de lui, et qu'il assume ses responsabilités, conscient du rôle important qu'il a à jouer dans le système de santé. La politique en matière de santé

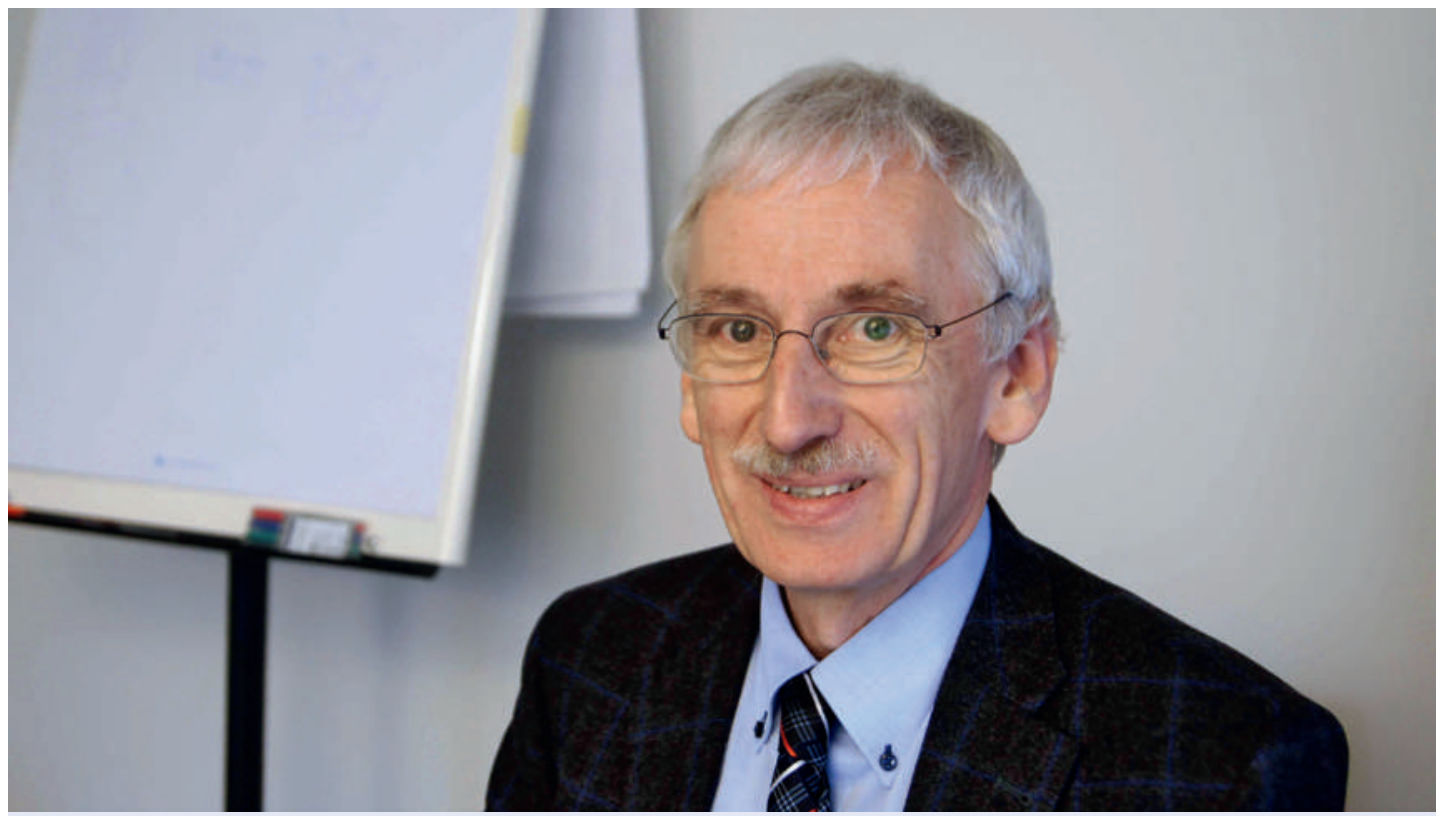

Peter Wiedersheim, le nouveau président de l'Assemblée des délégués de la FMH, entend mieux intégrer les membres dans la politique de santé. 


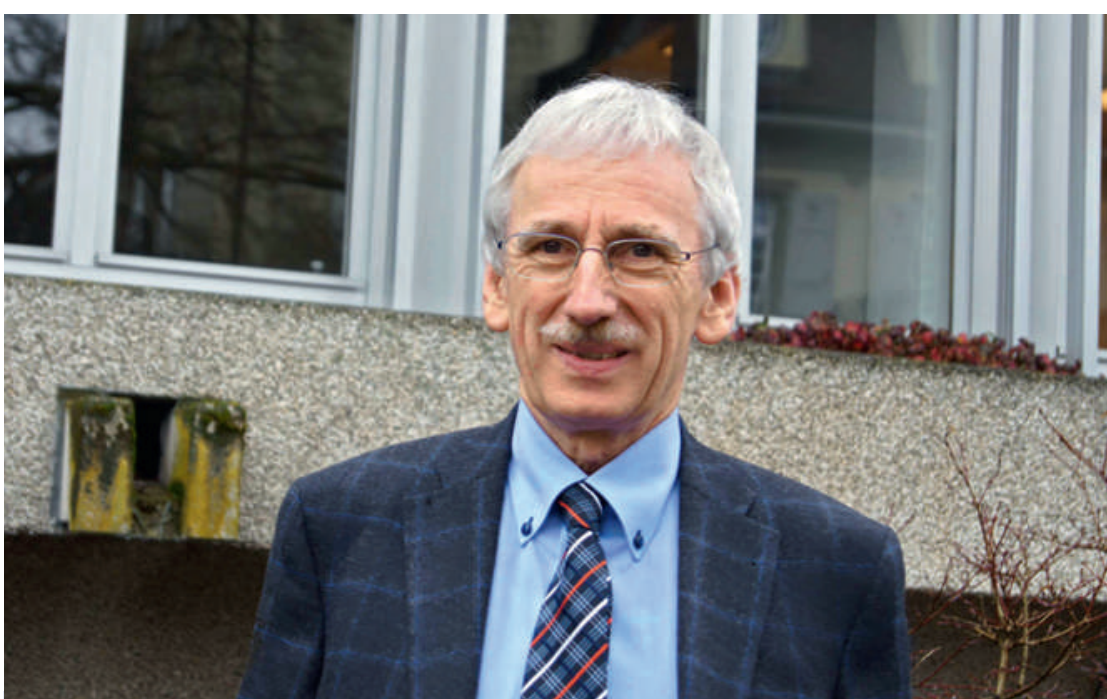

«En tant qu'acteur du système de santé de notre pays, nous devons jouer un rôle actif dans l'élaboration de l'agenda et anticiper.»

menée par les différents organes de la FMH doit avoir un impact plus important tant à l'interne qu'à l'externe. Je souhaite que les membres et plus particulièrement les jeunes médecins soient mieux intégrés dans les structures de direction de la FMH. Cela exige un meilleur flux d'informations entre la base et les organes de direction et vice-versa. Il me tient à cœur que les diversités linguistiques et culturelles du corps médical puissent pleinement exister au sein de notre assemblée. Il ne faut d'ailleurs pas oublier que pour pouvoir défendre au mieux nos intérêts, nous devons nous montrer unis face à l'extérieur. Mais dans l'ensemble, je souhaite contribuer à ce que le corps médical soit confiant car nous sommes les partenaires compétents pour toute question relative à la santé.

\section{«Le corps médical doit gagner en assurance, c'est-à-dire} qu'il doit également assumer une plus grande responsabilité à tous les niveaux.»

Quels seront selon vous les sujets prioritaires ces prochains mois?

A brève échéance, les sujets sont déjà en grande partie définis, pour ne pas dire «dictés». Nous abordons de manière beaucoup trop réactive les multiples tâches qui nous incombent au point que cela devient quasiment systématique! Si nous voulons agir en tant que principal protagoniste, nous devons jouer un rôle actif dans l'élaboration de l'agenda et anticiper. Mes thèmes de prédilection sont notamment améliorer les bénéfices pour les patients en tenant compte des coûts économiques globaux, battre en brèche les considérations sectorielles sur les coûts et ainsi améliorer la transparence. Il est également important de mettre en place une bonne recherche sur les soins et d'améliorer encore la qualité de ces derniers et de la prise en charge. Comme dit l'adage, celui qui a renoncé à devenir meilleur cesse d'être bon. Et précisément, fidèles à la devise «Fais du bon travail et fais-le savoir», nous devons communiquer mieux et plus souvent sur notre engagement en faveur de la qualité.

Qu'attendez-vous des membres de l'Assemblée des délégués?

De l'expérience en matière de direction, un bon ancrage en politique associative et un lien optimal et bidirectionnel entre la base et les organes de direction. Voilà mon souhait. Il va de soi que je continue aussi à apprécier l'excellent travail d'équipe et l'esprit collégial qui règnent à l'AD.

\section{«Celui qui a renoncé à devenir meilleur cesse d'être bon!»}

\section{Et comment concevez-vous la collaboration avec} le Comité central?

En plus des très bons contacts personnels que je noue depuis de nombreuses années avec les membres du Comité central, je participerai, à l'instar de mon prédécesseur, aux séances du Comité central. Cela me fournira un excellent aperçu des sujets d'actualité et des travaux en cours, et ce même si je ne participerai pas forcément aux procédures de prise de décision qui se font fréquemment au cours des séances à huis clos. La diversité des tâches que j'assume en politique

Peter Wiedersheim a été élu à la présidence de l'Assemblée des délégués à la fin 2013, succédant ainsi à Thomas Heuberger. Né en 1953 , il a passé son examen fédéral de médecin en 1978 et obtenu le titre de spécialiste en médecine interne générale en 1984 et, deux ans plus tard, le titre de spécialiste en rhumatologie. Après avoir exercé plusieurs années en milieu hospitalier, il a ouvert son propre cabinet devenu ensuite un cabinet de groupe. Parallèlement à son activité médicale, Peter Wiedersheim est engagé en politique professionnelle. Cela fait douze ans qu'il est président de la Société des médecins du canton de St-Gall. En 2007, il a accédé à la présidence de la Conférence des sociétés médicales de Suisse orientale et depuis 2012, il co-préside la Conférence des sociétés cantonales de médecine. En plus de ses différents mandats en politique professionnelle, il a obtenu, à la Haute école privée de gestion (PHW) Berne St-Gall Zurich, un diplôme de Manager médical et, l'année suivante, un Executive MBA dans la même école. 


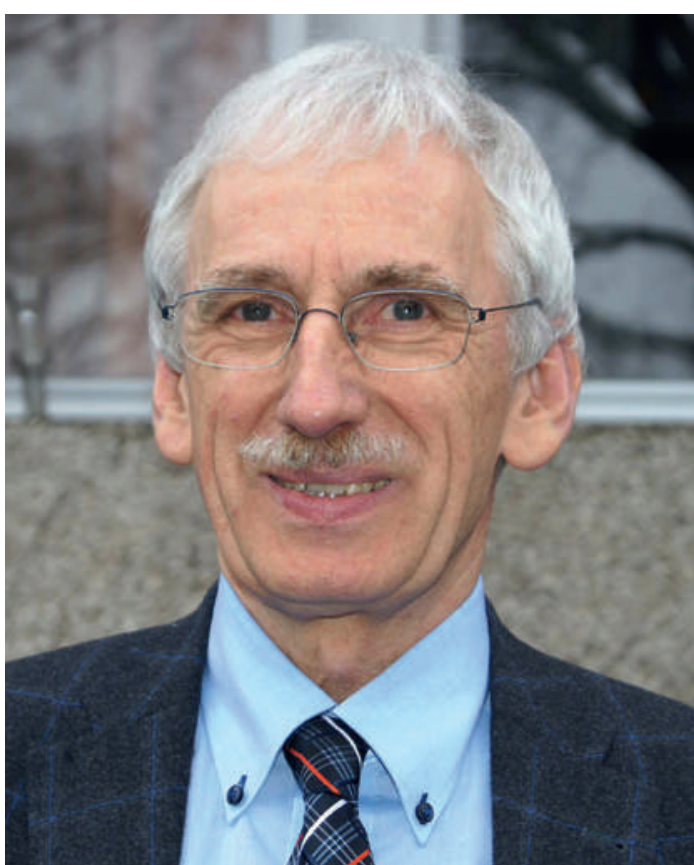

Pour Peter Wiedersheim, le flux d'informations entre les membres et les organes de direction revêt une importance majeure, au-delà des barrières linguistiques.

professionnelle donne lieu à de fréquents recoupements avec celles du Comité central et du Secrétariat général de la FMH. La très bonne collaboration et l'intense échange d'informations me sont très utiles dans mon activité à l'Assemblée des délégués.
Fondée en 2007, l'Assemblée des délégués de la FMH assure le lien entre le Comité central et la Chambre médicale. Elle a été conçue pour remplacer I'ancienne Conférence des présidents et ses quelque 100 membres dans le but de disposer d'un organe plus petit et donc mieux à même de participer activement à la politique de santé. Avec ses 33 représentants des organisations faîtières confirmés par la Chambre médicale, l'Assemblée des délégués examine de manière autonome toutes les questions importantes en matière de politique de santé et professionnelle et les adopte à l'intention de la Chambre médicale. Par ailleurs, elle définit la ligne politique suivie par la FMH lors de votations populaires, du lancement d'une initiative ou d'un référendum.

Les conflits d'intérêts sont toujours générés quand on poursuit des objectifs différents. Mon but avéré est d'œuvrer pour le bien des patients et ce peu importe ma casquette politique. Dans notre cabinet médical, cela signifie concrètement: «Notre objectif, c'est votre santé.» En politique professionnelle et corporative, il s'agit de garder constamment l'accent sur le bénéfice pour le patient, car mieux nous assumerons cette tâche, mieux nous nous porterons, non seulement nous mais aussi tout le monde.

Hormis vos différents mandats politiques, vous exercez également la médecine interne générale et la rhumatologie dans un cabinet de groupe. Quel est votre secret pour concilier vie professionnelle et vie privée?

\section{Sujets}

d'actualité

\section{du forum}

Venez débattre avec nous! Dans la rubrique forum, nous présentons régulièrement des sujets d'actualité politique, économique et scientifique ayant trait au système de santé. Donnez votre avis ou commentez les affirmations de vos confrères. Pour accéder au forum: www.bullmed.ch/forum/

\section{L'échange d'informations entre la base et les organes de la FMH doit être optimisé de manière bidirectionnelle.}

Je suis néanmoins conscient que selon le Règlement d'exécution de la FMH, l'Assemblée des délégués peut également faire office d'organe correcteur au sens d'une séparation des pouvoirs entre l'exécutif et la base du corps médical.

Vous co-présidez la Conférence des sociétés cantonales de médecine et présidez la Société des médecins du canton de Saint-Gall. Vous avez donc plusieurs casquettes politiques, cela n'engendre-t-il pas des conflits d'intérêts ou y voyez-vous plutôt des synergies pour l'Assemblée des délégués?
J'ai appris à décompresser même lors de courts instants et je suis également capable de me réjouir des choses simples du quotidien. Ma famille est mon ancrage. Elle fait non seulement preuve d'une grande compréhension à l'égard de mes nombreuses activités, mais me donne également la force et le soutien dont j'ai besoin. Mon père, âgé de 94 ans, lit encore quotidiennement le journal et m'envoie tous les articles relatifs à la politique de la santé. Mais le pilier principal, c'est ma femme. Cela fait 35 ans que je suis marié à une femme formidable, un véritable ange, qui me donne littéralement des ailes! 